The series ends with a lecture by Wigglesworth, osten. sibly on the hormonal regulation of differentiation in insects, but in fact covering a much wider field. It is, as one would expect, excellent, and good reading for anyone.

Perhaps the most valuable aspect of the book is the very real impression it conveys of the common ground that exists in the field of development in the botanical and zoological realms. It stimulates for this reason, and because it shows how much remains to be done; disappoints because of the first article, and a certain lack of depth; tantalizes, because one feels that an answer should be just around the corner. It would be improved by an index.

Michael BegG

\section{ANTIBODIES AND THEIR BIOSYNTHESIS}

Molecular and Cellular Basis of Antibody Formation Edited by J. Sterzl. (Proceedings of a Symposium held in Prague, June 1-5, 1964.) Pp. 683. (Prague : Publishing House of the Czechoslovak Academy of Sciences; New York and London: Academic Press, 1965.) \$20.

THE mechanism of antibody formation is one of the outstanding problems of biology, posing basic questions to all the many branches of science which contribute to molecular biology and the study of cell specialization. When most of the world's outstanding workers are brought together for a symposium on this subject, the resulting publication must be of interest to all who are concerned with immunology.

The report presents the entire symposium with the introductory lectures, followed by the main subject matter divided into five groups. The first is concerned with the nature of immunogenicity. The second group, on the characterization of antibodies, is subdivided into sections on the structure of the antibody molecule and antibody combining site, on the genetic determination of the peptide chains of antibodies and on the heterogeneity of antibodies. The third group, on the inductive phase and primary response in antibody formation, is subdivided into three sections describing the onset of antibody formation during development, the onset and dynamics of antibody formation studied with isolated cells, and the role of nucleic acids in antibody formation. The fourth group deals with the nature of the secondary response in antibody formation and the effects on this of ionizing radiation. The report closes with two papers and a general discussion on theories of the mechanism of antibody formation.

Many of the papers are presented in conventional journal form with substantial experimental detail, but others are more discursive and less well documented. There is also considerable variation in the length and clarity of presentation; one paper does not include the illustrations which the discussion indicates were shown as slides at the symposium. With so many contributions covering such a specialized subject there is naturally considerable overlap, but this contributes to the continuity of the subject matter. The detailed discussions which appear at the end of each paper are of little value. 'The more general discussions at the end of each section, howover, serve a useful purpose in allowing the presenta. tion of results from preliminary experiments as well as providing a forum for the further examination and correlation of points of interest raised in the individual papers.

One naturally compares the report of this symposium with its predecessor describing the 1959 symposium which was also held under the auspices of the Czechoslovak Academy of Sciences. That the size has almost doubled is a clear indication of the increasing interest in immunology. It is perhaps particularly relevant that the main increase has occurred in the field of immunoglobulin structure which has now achieved the status of having an internationally accepted nomenclature. The proposals for immunoglobulin terminology are included in the 1964 report and it is somewhat disappointing to find that in the interval before publication it was not possible to incorporate the new nomenclature in the presented papers.

One is left with the final questions. What purpose does publication of such a symposium serve and to what extent is this achieved in the present volume? Many symposium contributions can be found in standard journals in almost identical form. This symposium is no exception but perhaps can claim that it has drawn together in one place information which is scattered over many diverse journals. As a balanced view of the accumulated evidence and current hypotheses on antibody formation it is quite successful, although its large size provides rather too much reading even for the enthusiast. The rapid advance in immunological research has meant that the delay of about two years in publication has detracted from the significance of some of the work. It can, however, be recommended as presenting a much more integrated and advanced view of the subject than would have appeared possible five years previously.

J. E. FOTHERGILI

\section{NOBLE GAS ANAESTHETICS}

\section{The Aetiology of Compressed Air Intoxication and Inert Gas Narcosis}

By P. B. Bennett. (International Series of Monographs in Pure and Applied Biology. Division: Zoology, Vol. 31.) Pp. xvi +116. (London and New York: Pergamon Press, Ltd., 1966.) 35s. net.

OF all the sagas of physiological exploration, few can equal the investigation of myoneural transmission as a demonstration both of the rewards which follow the marriage of experimental method to hypothesis, and of the almost cathartic effect of uniting apparently opposing theories. First there was Claude Bernard's classical experimental proof of a junctional area, then came the work of Dale, Feldberg, Brown, Voigt and many others directed towards a humoral transmission theory, and a little later that of Eccles, Katz and Kuffler emphasizing the electrical phenomena of transmission, and so to the final proposition of the present theory bringing these two aspects together. This has been a story of dogged investigation based on hypothesis and the experimental proof or disproof thereof. The final dénouement had to await Hodgkin's fine exploration of demarcation potentials and their derivation from ionic asymmetry about the plasma membrane and the elegant proof that the potential prodicted from observed ionic migrations during the stimulus transmission equalled that found by experimental measurement.

A similar catalyst is necessary to bring together all the various aspects of the actions of inert gases on the central nervous synapses. Few will question that there is a missing link in the story, and the quest for it offers one of the great challenges to contemporary pharmacological investigation. So far, all the various theories accounting for the actions of anaesthetics on the central nervous system have been derived from those physical properties of the inert substances which appear to bear a direct relationship to their potency as central nervous depressants. These theories have, for example, been based on the solubility of the agent in lipids (Meyer and Overton), their ability to form hydrates in a microcrystalline form and so increase the impedance of nerve tissue (Pauling). their capacity to produce a reversal of oil in water emulsions and so possibly stabilize plasma membranes and the possible relationship of narcotic action to Van de Waal's attractions (Featherstone and Muehbecker). Whatever 